\title{
Hiperemesis por cannabis vs vómitos cíclicos
}

\section{Cannabinoid hyperemesis syndrome versus cyclic vomiting syndrome}

\author{
Guillermo Burillo-Putze *, Iván Hernández-Ramos**, Manuel Isorna-Folgar***. \\ * Departamento de Medicina Física y Farmacología, Universidad de La Laguna. \\ ** Servicio de Urgencias, Hospital Universitario de Canarias, Tenerife, España. \\ *** Grupo EVICT, Universidad de Vigo, España.
}

$\mathrm{E}$ 1 síndrome de hiperemesis cannabinoide (SHC) es todavía bastante desconocido entre los clínicos españoles de cualquier especialidad, como el trabajo de Ochoa-Mangado y Madoz-Gúrpide (2021) pone de manifiesto y al que nos gustaría aportar otros aspectos a tener en cuenta.

El SHC se incluyó en 2016 en la clasificación de trastornos funcionales digestivos (Clasificación Roma IV), donde se incorporó en el grupo de trastornos funcionales gastrointestinales, apartado B3 (trastornos de náuseas y vómitos), junto al síndrome de vómitos cíclicos (SVC). Básicamente el SHC se diferencia del SVC en que éste último suele asociarse a migrañas frecuentes, patología psiquiátrica concomitante, vaciamiento gástrico rápido y ausencia de consumo de cannabis. No obstante, algunos autores han encontrado hasta un $30 \%$ de consumo de cannabis en pacientes con diagnóstico de SVC, el cual podría deberse a las propiedades antieméticas del cannabis (Drossman y Hasler, 2016; Spiller, Künzler y Caduff, 2019).

La prevalencia en España podría estar en torno al $18 \%$ entre los consumidores crónicos de cannabis (Narváez et al., 2016), mientras que cálculos recientes realizados en USA indican que podría llegar al $33 \%$ en consumidores problemáticos (Habboushe, Rubin, Liu y Hoffman, 2018).
Según EDADES 2019/20, la presencia de consumo problemático de cannabis entre los 15 y los 64 años es del 1,9\%, unas 590.000 personas (Delegación del Gobierno para Plan Nacional sobre Drogas., 2021). A este reservorio de potenciales pacientes en riesgo de presentar SHC, hay que añadir el aumento de la potencia de las variedades de cannabis usadas en la actualidad, así como al mayor consumo de cannabinoides sintéticos (en cuyos usuarios también se han observado casos de SHC) (Bick, Szostek y Mangan, 2014). Todo ello augura, en nuestra opinión, un incremento de posibles casos de SHC, a tener en cuenta por los profesionales sanitarios (Galicia, 2020). En este sentido, el SHC puede presentarse con cuadros clínicos que precisan actuación urgente, como neumotórax o neumomediastino (Hernández Ramos, Parra Esquivel, López-Hernández y Burillo-Putze, 2019), e incluso recientemente se ha publicado una serie en la que se relaciona el SHC con muertes (Nourbakhsh, Miller, Gofton, Jones y Adeagbo, 2019).

Mención aparte merece el consumo de cannabis por embarazadas y la posibilidad de desarrollar un SHC, debiendo considerarlo en la anamnesis como causa de vómitos, para no interpretarlo erróneamente como hiperemesis gravídica. Además, el abuso de duchas de agua caliente de forma prolongada durante el primer trimestre de la gestación se

Recibido: Marzo 2021; Aceptado: Abril 2021.

Enviar correspondencia a: Dr Guillermo Burillo-Putze. Departamento de Medicina Física y Farmacología. Facultad de Ciencias de la Salud. Universidad de La Laguna. Carretera La Cuesta-Taco, s/n, La Laguna, 38320, Tenerife, España.

E-mail: gburillo@telefonica.net 
ha asociado con defectos del tubo neural, atresia esofágica, onfalocele y gastrosquisis, así como un incremento del riesgo de caídas en gestantes. (Abreu Jáuregui, López Hernández, Mendoza Romero y Armas Pérez, 2020).

En cuanto al tratamiento del SHC, sólo se han publicado 2 ensayos clínicos hasta la fecha, ambos en 2020, comparando haloperidol frente a ondansetrón (Ruberto et al., 2021) y capsaicina frente a placebo (Dean et al., 2020), con una treintena de pacientes en cada uno. Ambos fármacos han demostrado ser eficaces para el control de las náuseas, aunque son necesarios nuevos ensayos con un número mayor de pacientes y otros fármacos a priori útiles, como es el caso del droperidol (Lee, Greene y Wong, 2019).

En síntesis, creemos que es preciso una mayor sospecha diagnóstica del SHC en pacientes con náuseas como motivo de consulta que acudan a Urgencias u otros dispositivos sanitarios. Con ello podremos avanzar en conocer la verdadera incidencia de este síndrome en España, probablemente superior a la existente, como complicación del consumo crónico de cannabis.

\section{Referencias}

Abreu Jáuregui, E., López Hernández, Á., Mendoza Romero, C. L. y Armas Pérez, M. D. P. (2020). Cannabinoid hyperemesis syndrome during pregnancy: A case report. Atencion Primaria, 52, 513-514. doi:10.1016/j. aprim.2019.10.009.

Bick, B. L., Szostek, J. H. y Mangan, T. F. (2014). Synthetic cannabinoid leading to cannabinoid hyperemesis syndrome. Mayo Clinic Proceedings, 89, 1168-1169. doi:10.1016/j.mayocp.2014.06.013.

Dean, D. J., Sabagha, N., Rose, K., Weiss, A., France, J., Asmar, T.,... Miller, J. (2020). A pilot trial of topical capsaicin cream for treatment of cannabinoid hyperemesis dyndrome. Academic Emergency Medicine, 27, 1166-1172. doi:10.1111/acem.14062.

Delegación del Gobierno para Plan Nacional sobre Drogas. (2021). Encuesta sobre alcohol y otras drogas en España, EDADES 2019/20. Recuperado de https://pnsd. sanidad.gob.es/profesionales/sistemasInformacion/sistemaInformacion/pdf/2019-20_Informe_EDADES.pdf.

Drossman, D. A. y Hasler, W. L. (2016). Rome IV - Functional GI disorders: Disorders of gut-brain interaction. Gastroenterology, 150, 1257-1261. doi:10.1053/j.gastro.2016.03.035.

Galicia, M. (2020). Efectos adversos agudos en usuarios de drogas sintéticas: Una aproximación a la magnitud del problema en España. Emergencias, 32, 7-8.

Habboushe, J., Rubin, A., Liu, H. y Hoffman, R. S. (2018). The prevalence of cannabinoid hyperemesis syndrome among regular marijuana smokers in an urban public hospital. Basic E Clinical Pharmacology E Toxicology, 122, 660-662. doi:10.1111/bcpt.12962.
Hernández Ramos, I., Parra Esquivel, P., López-Hernández y Burillo-Putze, G. (2019). Neumomediastino espontáneo de repetición secundario al síndrome de hiperémesis por cannabis. Anales del Sistema Sanitario de Navarra, 42, 227-230. doi:10.23938/ASSN.0635.

Lee, C., Greene, S. L. y Wong, A. (2019). The utility of droperidol in the treatment of cannabinoid hyperemesis syndrome. Clinical Toxicology, 57, 773-777. doi:10.1080/1 5563650.2018 .1564324$.

Narváez, C. C., Gilbert, M. M., De Santiago, E. B., Farreres, J. B., Serven, E. G. y Crespillo, J. C. (2016). Síndrome de hiperemesis cannabinoide. Reporte de seis nuevos casos clínicos y resumen de casos previos publicados. Adicciones, 28, 90-98. doi:10.20882/adicciones.776.

Nourbakhsh, M., Miller, A., Gofton, J., Jones, G. y Adeagbo, B. (2019). Cannabinoid hyperemesis syndrome: Reports of fatal cases. Journal of Forensic Sciences, 64, 270274. doi:10.1111/1556-4029.13819.

Ochoa-Mangado, E. y Madoz-Gúrpide, A. (2021). Cannabis use and cyclic vomiting. Adicciones, 33, 75-76. doi:10.20882/adicciones. 1581.

Ruberto, A. J., Sivilotti, M. L. A., Forrester, S., Hall, A. K., Crawford, F. M. y Day, A. G. (2021). Intravenous haloperidol versus ondansetron for cannabis hyperemesis syndrome (HaVOC): A randomized, controlled trial. Annals of Emergency Medicine, 77, 613-619. doi:10.1016/j. annemergmed.2020.08.021.

Spiller, T. R., Künzler, K. y Caduff, B. (2019). Cyclic vomiting syndrome: An important differential diagnosis of cannabinoid hyperemesis syndrome. BMJ, 366, 15615. doi:10.1136/bmj.15615. 